Compton scattering on stable targets of arbitrary spin

Pais, A, Ghani, A, Salmeron, R A

Published in Nuovo Cimento 53 (1968) 433-454

This article has been scanned with the kind permission of the Società Italiana di Fisica.

From: Angela Oleandri <oleandriesif. it>
Date: october 12, 2007 4:07:47 PM GMT+02:00

To: Salvatore Mele <Salvatore. Melęcern.ch>
subject: Re: Reguest for permission to reproduce published material.

Dear Dr Mele,

further to your request, sent in July to Carmen Vasini (herewith attached)

I'm pleased to inform you that the Italian physical Society is happy

grant you permission of reproduct 


\title{
Compton Scattering on Stable Targets of Arbitrary Spin.
}

\author{
A. PAIS $\left(^{*}\right)$ \\ OERN - Geneva \\ (ricevuto il 16 Agosto 1967)
}

\begin{abstract}
Summary. - For Compton scattering on stable particles with spin $\mathbb{S}$ there exists, apart from the Thomson and the $(g-2)$ sum rules, a set of $(2 S-1)$ total-cross-section sum rules (for $S \geqslant 1$ ) which, in unsubtracted form, are superconvergence relations. Applied to nuclei, the possible validity of this no-subtraction form can be related to asymptotic additivity. Low-energy theorems are proved to exist for all $2 S+1$ intrinsic multipole moments. The case $S=1$ is treated in some detail.
\end{abstract}

\section{1. - Introduction.}

It is the purpose of this paper to discuss a number of low-energy theorems for Compton scattering on a stable target of arbitrary spin $S$. Some of the results have already been stated in a preceding note $\left(^{1}\right)$. The assumptions made are the same as in the earlier treatments $\left(^{2,3}\right)$ for $S=\frac{1}{2}$. In particular, $P$ and $T$ noninvariant effects are neglected. Moreover the target is assumed to be nondegenerate with any other particle or continuum state with the same mass and identical other quantum numbers $\left({ }^{4}\right)$. The results are exact in the strong interactions. We come back in Sect. 3 to the question to what extent higher-order electromagnetic interactions can also be incorporated.

Throughout this paper we shall work in the laboratory system, for definiteness. Whenever we deal with polarized targets, the target spin shall be quan-

(*) Permanent address: Rockefeller University, New York, New York.

(1) A. PaIS: Phys. Rev. Lett., 19, 544 (1967).

(2) F. E. Low: Phys. Rev., 96, 1428 (1954).

(3) M. Gril-MaNn and M. L. Goldberger: Phys. Rev., 96, 1433 (1954).

$\left.{ }^{4}\right)$ The theorems to be stated nevertheless apply to a particle like the $\Sigma^{+}$as long as one neglects the weak interactions. 
tized along the direction of the incoming beam, which is taken to be the threedirection. For photons, the transverse radiation gauge will be used.

The Iow-energy theorems to be derived fall into two categories.

1'1 Total cross-section theorems. - As the case may be, "total " cross-sections may refer to situations in which either or not the photon and/or the target is polarized, buit in which no other observable (total angular momentum, parity) is projected out. There are $2 S+1$. total cross-section theorems; they are discussed in Sect. 2. They are based, in part, on some low-energy theorems of a simple kind which are also stated in Sect. 2 , and which are proved in sect. 4 and 5. Apart from low-energy theorems, the only other ingredient needed for the total cross-section theorems are assumptions on high-energy behaviour. The complete set of total cross-section sum rules are: the "Thomson scattering sum rule" which needs at least one subtraction; the " $g-2$ " sum rule, eq. (2.22) below; a set of sum rules (for $S \geqslant 1$ ) involving only unpolarized photons on polarized targets, eq. (2.13) below; and at set of sum rules (for $S \geqslant \frac{3}{2}$ ) involving polarized photons on polarized targets, eq. (2.25) below.

Warning. It is not rigorously known, for any of the eqs. (2.22), (2.13), (2.25) whether it is true or not that no subtractions are needed. (For eq. (2.22) and $S=\frac{1}{2}$, the no-subtraction ansatz looks promising, however.) The no-subtraction form of these equations, as given in Sect. 2, must therefore be considered rather as tests for high-energy behaviour, as is spelled out in more detail in Sect. 2. The easiest relations to test from an experimental point of view are clearly those given in eq. (2.13). Such relations applied to nuclei (with spin $\geqslant \frac{1}{2}$ ) might nevertheless contain information on the photon-nucleon interaction, because at high energies the interaction with the nucleus should presumably be well approximated by coherent nucleon .effects, with appropriate spin correlations. In fact, asymptotic additivity in the nucleon amplitudes is a sufficient argument for the sum rules (2.13) and (2.25) to be true. If there is such additivity, the rate of saturation of these rules may give information as to how soon such additivity sets in.

The possible need for subtractions in these relations does of course not change their number. In any event, therefore, a larger number of total crosssection sum rules than the number $2 S+i$ given here can exist only if more detailed dynamical assumptions are made.

1:2. Intrinsic multipole moment theorems. - A particle of spin $S$ has $2 S+1$ intrinsic multipole moments (which may or may not be zero). The existence of low-energy theorems for the total electric charge and for the magnetic moment (or, more precisely, as we shall see, for $g-2$ where $g$ is the gyromagnetic ratio) has long been known $\left(^{2 \cdot 3}\right)$. It has been pointed out ( $\left.{ }^{1}\right)$ that there exist low-energy theorems for all intrinsic multipole moments. The 
detailed proof will be given in Sect. 4 for the quadrupole moment and in Sect. 5 for the general case.

After the analysis of the total cross-section questions in sect. 2 , the general formalism needed for the derivation of low-energy theorems is discussed in Sect. 3. There, a simple explicit construction is given of a complete and linearly independent set of amplitudes in terms of which one can parametrize the general amplitude for the scattering of a vector meson (with or without transversality imposed) on a target with arbitrary $S$, (construction of the "complete minimal basis "). It should be stressed that, for such questions, the use of an explicitly covariant formalism with its host of auxiliary constraints, is often an impediment rather than a help $\left({ }^{5}\right)$. Without loss of physical covariance we shall avoid such formalisms. Spin will only appear via the three-vector spin operator $S$ which satisfies $\left[S_{1}^{r}, S_{2}\right]=i S_{3}$, etc. Nor is one thereby bound to any particular frame of reference.

The method followed in this paper is the one of Low $\left({ }^{2}\right)$. Apart from the construction of the complete minimal basis, the only ingredients needed are: the determination of the pole-term contributions by eqs. (3.5)-(3.8) below; and Singh's lemma, eq. (3.12) below.

\section{2. - Total cross-section theorems.}

Let us first consider the properties of the Compton amplitudes at strictly zero frequency. In that case there are only three independent reduced matrix elements, namely for total spin $S+1, S, S-1$ (for $S=\frac{1}{2}$ there are only two, for $S=0$ only one). Correspondingly the most general form of the zero-energy amplitude matrix $\left({ }^{6}\right)$ is

$$
A(0)=A_{1}(0) \boldsymbol{\epsilon}^{\prime} \boldsymbol{\epsilon}+A_{2}(0) \boldsymbol{S}\left(\boldsymbol{\epsilon}^{\prime} \times \boldsymbol{\epsilon}\right)+A_{3}(0)\left\{\boldsymbol{S} \boldsymbol{\epsilon}, \boldsymbol{S} \boldsymbol{\epsilon}^{\prime}\right\},
$$

with three parameters.

Let $a\left(h, S_{3}, \omega\right)$ denote the elastic forward scattering amplitude for a photon with frequency $\omega$ and helicity $h$ on a target with polarization $S_{3}$. The threshold amplitudes $a\left(h, S_{3}, 0\right)$ can be expressed in terms of the $A_{i}(0)$ as follows:

$$
a\left( \pm 1, S_{3}, 0\right)=A_{1}(0) \pm i A_{2}(0) S_{3}+A_{3}(0)\left(S^{2}-S_{3}^{2}\right)
$$

Note that, for $S \geqslant 1$, there exist also «inelastic » forward scattering amplitudes, namely double spin-flip amplitudes corresponding to $\left|\Delta S_{3}\right|=2$ between initial

$\left(^{5}\right)$ See also S. Weinberg: Phys. Rev., 133, B 1318 (1964).

${ }^{(6)} A(0)$ is a matrix with respect to target spin. Hereafter we shall often speak of amplitudes instead of amplitude matrices whenever the meaning is obvious. Notations: $\{a, b\}=a b+b a$. $\boldsymbol{\epsilon}$ and $\boldsymbol{\epsilon}^{\prime}$ are the initial and final photon polarization vectors respectively, $\hbar=\dot{c}=1$. 
and final states. These amplitudes are proportional to $A_{3}(0)$. The values of the $A_{i}(0)$ are as follows:

1)

$$
A_{1}(0)=-\frac{e^{2}}{M}
$$

where $e$ and $M$ are the target charge and mass. This is the "Thomson limit" result.

Remark. In this paper, all amplitudes are normalized such that the optical theorem reads

$$
\operatorname{Im} a\left(h, S_{3}, \omega\right)=\omega \sigma\left(h, S_{3}, \omega\right)
$$

where $\sigma\left(h, S_{3}, \omega\right)$ is the corresponding total cross-section.

2)

$$
A_{2}(0)=0
$$

As is well known, this result follows essentially from crossing symmetry. 3)

$$
A_{3}(0)=0
$$

Equation (2.6) has in part been discussed previously $\left({ }^{1}\right)$. Its derivation is given in Sect. 4 and 5 below. Equations $(2.3),(2.5),(2.6)$ are the three zero-energy theorems for Compton scattering for any $S$. Equation (2.6) implies in particular the vanishing of double spin-flip at zero energy.

Let us next ask what are all the total cross-section rules which can arise in Compton scattering. For this purpose, consider the complete amplitude $A(\omega, \theta, \phi)$, where $\omega$ is the initial photon frequency and $\theta, \phi$ denote the polar angles of the final photon momentum $\boldsymbol{k}^{t}$ relative to the initial momentum $\boldsymbol{k}$ (which lies in the three-direction). We make a multipole expansion of $A(\omega, \theta, \phi)$ :

$$
\dot{A}(\omega, \theta, \phi)=\varepsilon_{m}^{\prime} A_{m n}(\omega, \theta, \phi) \varepsilon_{n}=\frac{1}{M} \sum_{N L M}\left(\frac{\omega}{M}\right)^{N} \varepsilon_{m}^{\prime} A_{m n}^{(N L M)} \varepsilon_{n} T_{L M}(\theta, \phi)
$$

The quantities $A_{m n}^{(N L M)}$ are still matrices with respect to the target spin. In this language, eqs. (2.3), (2.5), (2.6) can be summarized as follows,

$$
A_{m n}^{(000)}=-e^{2} \delta_{m n} \sqrt{4 \pi}
$$

Sum rules involving total cross-sections are necessarily associated with $L=M=0$. Thus we must ask what can be said of the quantities $A_{m n}^{(N 00)}$. 
21. The "helieity nonflip" case. - Define an average forward amplitude with respect to photon helicity:

$$
2 a\left(S_{3}, \omega\right) \equiv a\left(1, S_{3}, \omega\right)+a\left(-1 ; S_{3}, \omega\right),
$$

and a corresponding total cross-section $\sigma\left(S_{3}, \omega\right)$ for unpolarized photons:

$$
\operatorname{Im} a\left(S_{3}, \omega\right)=\omega \sigma\left(S_{3}, \omega\right)
$$

The $a\left(S_{3}, \omega\right)$ satisfy the symmetry relation

$$
a\left(S_{3}, \omega\right)=a\left(-S_{3}, \omega\right)
$$

so that the number of independent $a\left(S_{3}, \omega\right)$ is equal (") to $[S+1]$.

The $a\left(S_{3}, \omega\right)$ are associated with $A_{m n}^{(N N 0)}$ with $N$ even. Their zero-energy behaviour is therefore given by eq. (2.8). The average $a(\omega)$ of $a\left(S_{3}, \omega\right)$ over $S_{3}$ yields the total cross-section $\sigma(\omega)$ for unpolarized photons on an unpolarized target. According to eq. (2.8), $\sigma(0)$ gives the Thomson limit for any spin. It is well known that the dispersion relation for $a(\omega)$ needs at least one subtraction $\left({ }^{2,3}\right)$.

Apart from $a(\omega)$, there remain $[S]$ independent quantities $a\left(S_{3}, \omega\right)$. For any $\omega$ these can be represented by

$$
a\left(S_{3}, \omega\right)=\alpha_{0}(\omega)+\alpha_{2}(\omega) S_{3}^{2}+\ldots+\alpha_{2[S]} S_{3}^{2[s]} .
$$

There are $[S+1]$ terms in this polynomial. But for $\omega=0$ there is only one term, according to eq. (2.6) or (2.8), which expresses polarization independence at threshold.

What is the asymptotic behaviour of $a\left(S_{3}, \omega\right)$ ? Surely, $a\left(S_{3}, \omega\right)$ does not go to zero, as the Thomson relation needs at least one subtraction. It is conceivable, however, that asymptotically $a\left(S_{3}, \omega\right)$ is independent of $S_{3}$. If and only if this is the case we can combine the low-energy theorem (2.8) with this asymptotic behaviour to get

$$
\int\left[\sigma\left(S_{3}, \omega\right)-\sigma\left(S_{3}^{\prime}, \omega\right)\right] \mathrm{d} \omega=0, \quad S_{3}, S_{3}^{\prime} \geqslant 0
$$

The question mark in front of this equation and similar equations to follow has reference to the warning given in Sect. 1. We have $\left(^{1}\right)$

(7) $[n]$ is the entire part of $n$. 
Theorem 1. The $[S]$ sum rules given in eq. (2.13) are a test for asymptotic polarization independence. That is to say, the complete set of these [S] relations is true if and only if $a\left(S_{3}, \omega\right)$ is asymptotically independent of $S_{3}$.

For $S=1, \frac{3}{2}$ we have a single relation:

$$
\begin{array}{ll}
\int[\sigma(1, \omega)-\sigma(0, \omega)] \mathrm{d} \omega=0, & S=1, \\
\int\left[\sigma\left(\frac{3}{2}, \omega\right)-\sigma\left(\frac{1}{2}, \omega\right)\right] \mathrm{d} \omega=0, & S=\frac{3}{2} .
\end{array}
$$

These relations depend crucially on the fact that $A_{3}(0)=0$. On the other hand, for $S \geqslant 2$ we would still have $[S-1]$ sum rules of the type eq. (2.13) independently of the value of $A_{3}(0)$, as long as we have asymptotic polarization independence. For example the relation

$$
\int[\sigma(2, \omega)-\sigma(1, \omega)] \mathrm{d} \omega=3 \int[\sigma(1, \omega)-\sigma(0, \omega)] \mathrm{d} \omega, \quad S=2,
$$

is independent of the value of $A_{3}(0)$. Thus, we see that of the [S] relations $(2.13)$ only one depends on the low-energy theorem (2.6). The remaining ones (for $S \geqslant 2$ ) depend on the general invariance properties and on asymptotic behaviour only. For the sake of completeness we note that, for $S \geqslant 2$ some but not all of the relations (2.13) may be true if the aisymptotic behaviour of $a\left(S_{3}, \omega\right)$ is polarization dependent, but not maximally so. For example, eq. (2.16) remains valid (using èq. (2.6)) if asymptotically $a\left(S_{3}, \omega\right) \sim \alpha_{0}(\omega)+\alpha_{2}(\omega) S_{3}^{2}$ for $S=2$, that is, if $\alpha_{1}(\omega) \sim 0$.

2'2. The "helicity flip》 case. - Define a forward amplitude

$$
a^{(-)}\left(S_{3}, \omega\right) \equiv a\left(1, S_{3}, \omega\right)-a\left(-1, S_{3}, \omega\right)
$$

and a corresponding total cross-section $\sigma^{(-)}\left(\mathcal{S}_{3}, \omega\right)$ for polarized photons:

$$
\operatorname{Im} a^{(-)}\left(S_{3}, \omega\right)=\omega \sigma^{(-)}\left(S_{3}, \omega\right)
$$

The $a^{(-)}\left(S_{3}, \omega\right)$ satisfy the symmetry relation

$$
a^{(-)}\left(S_{3}, \omega\right)=-a^{(-)}\left(-S_{3}, \omega\right)
$$

so that the number of independent $a^{(-)}\left(S_{3}, \omega\right)$ is equal to $\left[S+\frac{1}{2}\right]$.

The $a^{(-)}\left(S_{3}, \omega\right)$ are associated with $A_{m n}^{N 00}$ with $N$ odd. In Sect. 4 and 5 we shall see that

$$
A_{m n}^{100}=-\frac{i e^{2}}{4}(g-2)^{2} \varepsilon_{m n l} S_{l} \sqrt{4 \pi}
$$


Therefore, if

$$
\lim _{\omega \rightarrow \infty} a^{(-)}(S, \omega)=0
$$

we have

$$
\text { (?) } \quad \frac{\pi^{2} \alpha S}{M^{2}}(g-2)^{2}=\int \frac{\mathrm{d} \omega}{\omega} \sigma^{(-)}(S, \omega) \text {, }
$$

which is the form, for arbitrary $S$, of the well-known magnetic moment sum rule $\left({ }^{8}\right)$. Numerical estimates indicate $\left({ }^{8}\right)$ that the no-subtraction ansatz implied by eq. (2.21) may well be true for $S=\frac{1}{2}$. Of course, for this as for the other sum rules of this Section, the fact that no subtractions may be needed for one value of $S$ does not imply anything for another value of $S$.

There now remain $\left[S-\frac{1}{2}\right]$ independent quantities $a^{(-)}\left(S_{3}, \omega\right)$. For any $\omega$ we have

$$
a^{(-)}\left(S_{3}, \omega\right)=\alpha_{1}(\omega) S_{3}+\alpha_{3}(\omega) S_{3}^{3}+\ldots+\alpha_{2\left[S+\frac{1}{2}\right]-1} S_{3}^{2\left[S+\frac{1}{2}\right]-1}
$$

There are $\left[S+\frac{1}{2}\right]$ terms in this polynomial. It is conceivable that

$$
\lim _{\omega \rightarrow \infty} a^{(-)}\left(S_{3}, \omega\right)=0
$$

If and only if this is the case we have, along with eq. (2.22) the $\left[S-\frac{1}{2}\right]$ number of relations

$$
\text { (?) } \quad \int\left[S_{3} \sigma^{(-)}\left(S_{3}^{\prime}, \omega\right)-S_{3}^{\prime} \sigma^{(-)}\left(S_{3}, \omega\right)\right] \frac{d \omega}{\omega}=0
$$

Hence we have

Theorem 2. The sum rules (2.22) and (2.25) are valid if and only if eq. (2.24) is true.

The relations eq. (2.25) are relevant only to $S \geqslant \frac{3}{2}$. Just as was mentioned after eq. (2.16), some of the relations (2.25) may survive (for $S \geqslant \frac{5}{2}$ ) if some but not all of the $\alpha_{i}(\omega)$ in eq. (2.23) vanish asymptotically.

So far we have used only the $A_{m n}^{\text {(Noo) }}$ for $N=0$ and 1 . It is well known, however, that there do not exist $\left(^{2,3}\right)$ low-energy theorems for $N \geqslant 2$ and $L=M=0$ which are independent of the details of the dynamics. Therefore it follows that we now have found all possible total cross-section sum rules ("total " in the sense defined in Sect. 1) for Compton scattering which depend

(8) S. D. DRell and A. C. HxaRN: Phys. Rev. Lett., 16, 908 (1966); M. Hosoda and K. Yamamoto: Progr. Theor. Phys., 36, 425, 426 (1966); G. Konisi and K. YAMAмото: Progr. Theor. Phys., 37, 538 (1967); H. PAgecs: Phys. Rev., 158, 1566 (1967). 
on low-energy theorems and asymptotic behaviour only. They are: the Thomson rule, the $(g-2)$ rule, eq. (2.22) and the "superconvergence" relations (2.13) and (2.25).

\section{3. - General formalism.}

Compton scattering is described by an amplitude matrix $\left(^{6}\right) A_{\mu \nu}\left(k^{\prime}, k\right)$ which is a tensor with respect to the photon polarization indices $\mu$ (final) and $\nu$ (initial). The relations

$$
k_{\mu}^{\prime} A_{\mu \nu}\left(k^{\prime}, k\right)=A_{\nu \lambda}\left(k^{\prime}, k\right) k_{\lambda}=0
$$

are a consequence of gauge invariance. From (3.1) it follows that $\left({ }^{9}\right)$

$$
k_{m}^{\prime} A_{m n}\left(k^{\prime}, k\right) k_{n}=-\omega^{\prime} \omega A_{44}\left(k^{\prime}, k\right)
$$

In the transverse gauge:

$$
\boldsymbol{\epsilon} \boldsymbol{k}=\boldsymbol{\epsilon}^{\prime} \boldsymbol{k}^{\prime}=0
$$

the physical amplitude $A$ is

$$
A=\varepsilon_{m}^{\prime} A_{m n}\left(k^{\prime}, k\right) \varepsilon_{n} .
$$

$A_{44}$ is given by

$$
\begin{aligned}
(2 \pi)^{-6}\left[\frac{E(\boldsymbol{p}) E\left(\boldsymbol{p}^{\prime}\right)}{M^{2}}\right]^{\frac{1}{2}} A_{44}= \\
\quad=\frac{\left\langle\boldsymbol{p}^{\prime}|\varrho| s(\boldsymbol{p}+\boldsymbol{k})\right\rangle\langle s(\boldsymbol{p}+\boldsymbol{k})|\varrho| \boldsymbol{p}\rangle}{E(\boldsymbol{p})+\omega-s(\boldsymbol{p}+\boldsymbol{k})}+\frac{\left\langle\boldsymbol{p}^{\prime}|\varrho| s\left(\boldsymbol{p}-\boldsymbol{k}^{\prime}\right)\right\rangle\left\langle s\left(\boldsymbol{p}-\boldsymbol{k}^{\prime}\right)|\varrho| \boldsymbol{p}\right\rangle}{E(\boldsymbol{p})-\omega^{\prime}-s\left(\boldsymbol{p}-\boldsymbol{k}^{\prime}\right)} .
\end{aligned}
$$

Here the following notations have been used. $\varrho$ is the electric charge density operator: $|\boldsymbol{p}\rangle$ is the one-particle (target) state with three-momentum $\boldsymbol{p}$; $s(\boldsymbol{p}+\boldsymbol{k})$ is a general (on shell) intermediate state with total three-momentum $(\boldsymbol{p}+\boldsymbol{k}) ; \boldsymbol{E}(\boldsymbol{p})=\left(\boldsymbol{p}^{2}+M^{2}\right)^{\frac{1}{2}}$. In eq. (3.5) and similar equations to follow, overall normalizations of amplitudes have been chosen so that eq. (2.4) is true for any $s$. A summation over a complete set of states $s$ is implied.

Next, $A_{\mu \nu}$ is divided into two parts,

$$
A_{\mu \nu}\left(k^{\prime}, k\right)=D_{\mu \nu}\left(k^{\prime}, k\right)+E_{\mu \nu}\left(k^{\prime}, k\right)
$$

( $\left.{ }^{9}\right)$ The metric $k_{\mu}=\left(k_{i}, k_{4}\right)=\left(k_{i}, i \omega\right)$ will be used. 
$U_{\mu \nu}$ refers to the unexcited or one-particle (target) pole contribution. Thus in eq. (3.5) we have

$$
U_{44} \leftrightarrow|s(\boldsymbol{p}+\boldsymbol{k})\rangle \equiv|\boldsymbol{p}+\boldsymbol{k}\rangle ; \quad s\left(\boldsymbol{p}-\boldsymbol{k}^{\prime}\right) \equiv\left|\boldsymbol{p}-\boldsymbol{k}^{\prime}\right\rangle
$$

Similarly

$$
\begin{aligned}
& -(2 \pi)^{6}\left[\frac{E(\boldsymbol{p}) E\left(\boldsymbol{p}^{\prime}\right)}{M^{2}}\right] U_{m n}= \\
& =\frac{\left\langle\boldsymbol{p}^{\prime}\left|j_{m}\right| \boldsymbol{p}+\boldsymbol{k}\right\rangle\left\langle\boldsymbol{p}+\boldsymbol{k}\left|j_{n}\right| \boldsymbol{p}\right\rangle}{E(\boldsymbol{p})+\omega-E(\boldsymbol{p}+\boldsymbol{k})}+\frac{\left\langle\boldsymbol{p}^{\prime}\left|j_{n}\right| \boldsymbol{p}-\boldsymbol{k}^{\prime}\right\rangle\left\langle\boldsymbol{p}-\boldsymbol{k}^{\prime}\left|j_{m}\right| \boldsymbol{p}\right\rangle}{E(\boldsymbol{p})-\omega^{\prime}-E\left(\boldsymbol{p}-\boldsymbol{k}^{\prime}\right)}
\end{aligned}
$$

$E_{44}$ is given by the right-hand side of eq. (3.5) but with the summation over the states $s$ restricted by the exclusion of the one-particle state.

There exists no such general and simple explicit expression for $E_{m n}$ of the type of eqs. (3.5) or (3.8). Rather, following Low's procedure ( $\left.{ }^{2}\right) E_{m n}$ will be determined, as well as possible, from eq. (3.2) which may be written as

$$
k_{m}^{\prime} E_{m n} k_{n}=-\left(k_{m}^{\prime} U_{m n} k_{n}+\omega^{\prime} \omega U_{44}\right)-\omega^{\prime} \omega E_{44}
$$

The physical amplitude

$$
\begin{gathered}
A=U+E \\
U=\varepsilon_{m}^{\prime} U_{m n} \varepsilon_{n} ; \quad E=\varepsilon_{m}^{\prime} E_{m n} \varepsilon_{n},
\end{gathered}
$$

contains only the transverse part $E$ of $E_{m n}$. Nevertheless, if we want to use eq. (3.9) for a partial determination of $E_{m n}$ we must find a parametrization for $E_{m n}$ in which transversality is not imposed from the outset. We call this the problem of the complete basis for $E_{m n}$.

The extent to which $E_{m n}$ can be determined from eq. (3.9) depends on the knowledge of $E_{41}$, given by the excited state part of eq. (3.5). As is well known $\left({ }^{2}\right), E_{44}$ is $O\left(\omega^{2}\right)$ for small $\omega$. This statement has recently been cast in a more precise form by SINGH $\left(^{10}\right)$ who has shown that

$$
E_{44}=k_{m}^{\prime} k_{n}\left[\Lambda_{m n}\left(\omega^{\prime}, \boldsymbol{k}^{\prime} ; \omega, \boldsymbol{k}\right)+\Lambda_{n m}\left(-\omega,-\boldsymbol{k} ;-\omega^{\prime},-\boldsymbol{k}^{\prime}\right)\right]
$$

where $\Lambda_{m n}$ is a three-tensor of second rank and free of kinematical singularities. It may be noted that the derivation $\left({ }^{10}\right)$ of eq. (3.12) is evidently independent of the target spin; and that for $S \neq 0$, the $\Lambda_{m n}$ are to be considered as matrices in the target spin.

(10) V. Srvat: Phys. Rev. Lett., 19, 730 (1967). 
Remark. In regard to eq. (3.5), the question arises as to the convergence of the summation over the states $s$. Tied to this is the question of the existence of the $\Lambda_{m n}$ with its stated properties. It could be that the validity of eq. (3.12) is more general than that of eq. (3.5), as was stressed by J. S. BeLL, in a private discussion (*).

It will be seen in the subsequent Sections that the statement: $E_{44}=O\left(\omega^{2}\right)$ is sufficient to derive the low-energy theorems, eqs. (2.3), (2.6), (2.20). On the other hand, for the derivation of the higher-multipole theorems the more specific form, eq. (3.12) is decisive.

A remark is now in order about the influence of higher-order electromagnetic effects on the statements made up to this point. Equations (3.1)-(3.11) are all rigorously valid in the presence of strong and electromagnetic interactions. Thus, in particular, the electromagnetic vertices which appear in $U_{44}$ and $U_{m n}$ may be considered to be exact in all these interactions. However, due to the presence of target + soft photon states it is no longer possible to consider eq. (3.12) the most general form for $E_{44}$. See further ref. $\left(^{2,3}\right)$ and ref. (1), footnote $\left({ }^{4}\right)$. In any event, for hadron problems the neglect (which we shall make here) of higher-order electromagnetic effects will presumably not affect quantitatively the final results.

The remaining problem regarding the general formalism is the construction of the complete basis for $E_{m n}$. More explicitly, we write $E_{m n}$ as $H_{m n}\left(\boldsymbol{k}^{\prime}, \boldsymbol{k}, S\right)$, that is, we consider $E_{m n}$ as a $3 \times 3$ matrix with elements that are $(2 S+1) \times$ $\times(2 S+1)$ matrices. $P$ invariance implies that

$$
E_{m n}\left(\boldsymbol{J}^{\prime}, \boldsymbol{k}, S\right)=E_{m n}\left(-\boldsymbol{k}^{\prime},-\boldsymbol{k}, S\right)
$$

while $T$ invariance can be stated as

$$
E_{m n}\left(\boldsymbol{k}, \boldsymbol{k}^{\prime}, \boldsymbol{S}\right)=E_{n m}\left(-\boldsymbol{k},-\boldsymbol{k}^{\prime},-S^{\prime}\right) .
$$

From eqs. (3.13), (3.14) it follows that the number of independent matrix elements among the $(3(2 S+1))^{2}$ quantities $E_{m n}$ is

$$
N_{\rho}(S)=(4 S-[S]+2)(2 S+[S]+2)
$$

We repeat that this is the number of independent amplitudes without the transversality conditions (3.3). Thus $N_{\rho}(S)$ is the number of amplitudes for the scattering of a $\rho^{0}$-meson on a target with spin $S$. Furthermore the number

(*) Added in proofs: see J. S. BELL: Nuovo Cimento, 52 A, 935, 1967. 
$N_{\rho}(S)$ refers to the nonforward direction. The construction of the complete $N_{\mathrm{p}}(S)$ basis is done in the following steps.

Let $\mathbb{E}_{m n}\left(\boldsymbol{k}^{\prime}, \boldsymbol{k}\right)$ refer to the case $S=0$. If we ignore for a moment the constraints, eqs. (3.13), (3.14), there are nine such quantities, which satisfy

$$
\begin{aligned}
& E_{m n}\left(\boldsymbol{k}^{\prime}, \boldsymbol{k}\right)=\varepsilon_{P} E_{m n}\left(-\boldsymbol{k}^{\prime},-\boldsymbol{k}\right), \\
& E_{m n}\left(\boldsymbol{k}^{\prime}, \boldsymbol{k}\right)=\varepsilon_{r} E_{n m}\left(-\boldsymbol{k},-\boldsymbol{k}^{\prime}\right),
\end{aligned}
$$

which fall into four groups depending on whether $\varepsilon_{P}= \pm 1, \varepsilon_{T}= \pm 1$. Define

$$
\boldsymbol{K}=\boldsymbol{k}^{\prime}+\boldsymbol{k}, \quad \boldsymbol{Q}=\boldsymbol{k}^{\prime}-\boldsymbol{k}, \quad \boldsymbol{R}=\boldsymbol{k}^{\prime} \times \boldsymbol{k} .
$$

Then we have the following set of $E_{m n}\left(\boldsymbol{k}^{\prime}, \boldsymbol{k}\right)$ :

$$
\begin{array}{lll}
\varepsilon_{P}=\varepsilon_{T}=1: & \delta_{m n}, K_{m} K_{n}, Q_{m} Q_{n} ; & \varepsilon_{m n l} R_{l}, \\
\varepsilon_{P}=-\varepsilon_{T}=1: & K_{m} Q_{n}+K_{n} Q_{m}, & \\
\varepsilon_{P}=-\varepsilon_{T}=-1: & K_{m} R_{n}+K_{n} R_{n} ; & \varepsilon_{m n l} K_{l}, \\
\varepsilon_{P}=\varepsilon_{P}=-1: & Q_{m} R_{n}+Q_{n} R_{m} ; & \varepsilon_{m n l} Q_{l},
\end{array}
$$

corresponding to the decomposition $9=4+1+2+2$. The set of eq. (3.19) is the $N_{\rho}(0)=4$ basis for $S=0$.

For $S>0$, consider the three rotational invariants $S_{m} X_{m}\left(\boldsymbol{k}^{\prime}, \boldsymbol{k}\right)$ where $X_{m}\left(\boldsymbol{k}^{\prime}, \boldsymbol{k}\right)$ is either $K_{m}, Q_{m}$ or $R_{m}$. We can again consider the distinctions

$$
\begin{aligned}
& S_{m} X_{m}\left(\boldsymbol{k}^{\prime}, \boldsymbol{k}\right)=\varepsilon_{P} S_{m} X_{m}\left(-\boldsymbol{k}^{\prime},-\boldsymbol{k}\right) \\
& S_{m} X_{m}\left(\boldsymbol{k}^{\prime}, \boldsymbol{k}\right)=\varepsilon_{T} S_{m} X_{m}\left(-\boldsymbol{k},-\boldsymbol{k}^{\prime}\right)
\end{aligned}
$$

and have

$$
\begin{array}{ll}
\varepsilon_{P}=\varepsilon_{P}=1: & S_{m} R_{m}, \\
\varepsilon_{P}=-\varepsilon_{T}=-1: & S_{m} K_{m}, \\
\varepsilon_{P}=\varepsilon_{T}=-1: & S_{m} Q_{m} .
\end{array}
$$

With the help of eqs. (3.19)-(3.22) and (3.25)-(3.27) we can now build up all $E_{m n}\left(\boldsymbol{k}^{\prime}, \boldsymbol{k}, S^{\prime}\right)$ by the following procedure. Define

$$
\begin{aligned}
& a ! b ! e !\left\langle K^{a} Q^{b} R^{c}\right\rangle \equiv \\
& \quad \equiv\left(\sum_{P} S_{m_{1}} S_{m_{2}} \ldots S_{m_{a}} S_{n_{1}} \ldots S_{n_{b}} S_{l_{1}} \ldots S_{l_{c}}\right) K_{m_{1}} \ldots K_{m_{a}} Q_{n_{1}} \ldots Q_{n_{b}} R_{l_{1}} \ldots R_{l_{c}}
\end{aligned}
$$


where the summation is over all the permutations of the indices of the spin vectors. We can assign to each $\left\langle K^{a} Q^{b} R^{c}\right\rangle$ a set of $\left(\varepsilon_{P}, \varepsilon_{T}\right)$ by repeated use of eqs. (3.25)-(3.27). Multiply any member of the set (3.19)-(3.24), by some fixed $\left(\varepsilon_{P}, \varepsilon_{r}\right)$ with a $\left\langle K^{a} Q^{b} R^{c}\right\rangle$ which has the same $\left(\varepsilon_{P}, \varepsilon_{r}\right)$. Then, we have one of the basis functions of $E_{m n}\left(\boldsymbol{k}^{l}, \boldsymbol{k}, S\right)$ we are looking for. Observe the following.

1) For given spin $S$ :

$$
a+b+c \leqslant 2 S,
$$

because of the closure of the spin algebra for given $S$.

2) The complete basis so constructed for given $S$ is at the same time a partial basis for all higher $S$.

3) For the vectors $\boldsymbol{K}, \boldsymbol{Q}, \boldsymbol{R}$ we have the completeness relation

$$
\begin{aligned}
R_{m} R_{n}=\left[K^{2} Q^{2}-(\boldsymbol{K} \boldsymbol{Q})^{2}\right] \delta_{m n}- & \\
& -\frac{1}{4}\left[Q^{2} K_{m} K_{n}+K^{2} Q_{m} Q_{n}-\boldsymbol{K} Q\left(K_{m} Q_{n}+K_{n} \dot{Q}_{m}\right)\right]
\end{aligned}
$$

which shows that $\left\langle K^{2}\right\rangle,\left\langle Q^{2}\right\rangle$ and $\left\langle R^{2}\right\rangle$ are not independent. In fact we may replace $\left\langle R^{2}\right\rangle$ by 1 , the unit matrix in spin space. We must therefore restrict $c$. in eq. (3.28) by

$$
c \leqslant 1 .
$$

As an example we give the $\left\langle K^{a} Q^{b} R^{c}\right\rangle$ for $S \leqslant \frac{3}{2}$ :

$$
\begin{array}{ll}
\varepsilon_{P}=\varepsilon_{T}=1: & 1,\langle R\rangle,\left\langle K^{2}\right\rangle,\left\langle Q^{2}\right\rangle,\left\langle K^{2} R\right\rangle,\left\langle Q^{2} R\right\rangle, \\
\varepsilon_{P}=-\varepsilon_{T}=1: & \langle K Q\rangle,\langle K Q R\rangle, \\
\varepsilon_{P}=-\varepsilon_{T}=-1: & \langle K\rangle,\langle K R\rangle,\left\langle K^{3}\right\rangle,\left\langle K Q^{2}\right\rangle, \\
\varepsilon_{P}=\varepsilon_{T}=-1: & \langle Q\rangle,\langle Q R\rangle,\left\langle Q^{3}\right\rangle,\left\langle Q K^{2}\right\rangle,
\end{array}
$$

with the dimension check

$$
N_{\rho}\left(\frac{3}{2}\right)=42=4 \times 6+1 \times 2+2 \times 4+2 \times 4 .
$$

Going backward, to find the $N_{\rho}(1)=25$ basis for $S=1$, simply drop the cubic terms in eqs. (3.32)-(3.35), etc.

It may be stressed that the relative ease of finding bases for arbitrary spins stems from the fact that we have of course insisted on completeness; but not on orthogonality. 
However, we are not quite finished yet. While we now have a complete basis, this is not yet the complete minimal basis. By minimality the following is meant. We are looking for an expansion

$$
E_{m n}\left(\boldsymbol{k}^{\prime}, \boldsymbol{k}, \boldsymbol{S}\right)=\sum_{i} A_{i}\left(\boldsymbol{k}^{\prime}, \boldsymbol{k}\right) E_{m n}^{(i)}\left(\boldsymbol{k}^{\prime}, \boldsymbol{k}, \boldsymbol{S}\right),
$$

where $i=1, \ldots, N_{\rho}(S)$, to be inserted in eq. (3.9). The $E_{m n}^{(i)}$ are the set of basis functions, the $A_{i}$ are the corresponding "four-point form factors 》. It is evident that we would introduce spurious kinematic singularities in the $A_{i}$ if we work with a basis for the $E_{m n}^{(i)}$ which has, for some $i$, more powers of $\omega$ than is necessary. We must therefore eliminate these extra powers. Having done that, we will have the complete "minimal" basis.

The reduction to the minimal basis is also quite simple. First of all we may make use of eq. (3.30) for this purpose. Example: take $\varepsilon_{m n l} R_{l}$ from eq. (3.19) and multiply by $\boldsymbol{S} \boldsymbol{R}$ from eq. (3.25). This is an appropriate but nonminimal basis function. Equation (3.30) informs us that in this product we may make the substitution

$$
R_{m} R_{n} \rightarrow \delta_{m n}
$$

without loss of completeness. This then gives the minimal basis function $\varepsilon_{m n l} S_{l}$; ete.

A second and last identity sufficient for the reduction to the minimal basis is (for any $\boldsymbol{X}$ )

$$
(\boldsymbol{S} \boldsymbol{X}) R_{m}=\left(\boldsymbol{X} \boldsymbol{k}^{\prime}\right)(\boldsymbol{k} \times \boldsymbol{S})_{m}-(\boldsymbol{X} \boldsymbol{k})\left(\boldsymbol{k}^{\prime} \times \boldsymbol{S}\right)_{m}+X_{m} \boldsymbol{S}\left(\boldsymbol{k} \times \boldsymbol{k}^{\prime}\right) .
$$

With the help of eqs. (3.37), (3.38) and after a bit of linear combination one obtains the following minimal basis $E_{m n}^{(i)}$ for the case $S=1$.

$$
\left\{\begin{aligned}
i=1 & \delta_{m n}, \\
2 & \varepsilon_{m i n} S_{l}, \\
3 & \left\{S_{m}, S_{n}\right\} \\
4 & k_{m} k_{n}+k_{m}^{\prime} k_{n}^{\prime}, \\
5 & k_{m} k_{n}^{\prime}, \\
6 & k_{m}^{\prime} k_{n}, \\
7 & \delta_{m n} \boldsymbol{S}\left(\boldsymbol{k} \times \boldsymbol{k}^{\prime}\right) \\
8 & \varepsilon_{m n l}\left(k_{l}(\boldsymbol{S} \boldsymbol{k})+k_{l}^{\prime}\left(\boldsymbol{S} \boldsymbol{k}^{\prime}\right)\right) \\
\mathbf{9} & \varepsilon_{m n l}\left(k_{l}\left(\boldsymbol{S} \boldsymbol{k}^{\prime}\right)+k_{l}^{\prime}(\boldsymbol{S} \boldsymbol{k})\right)
\end{aligned}\right.
$$




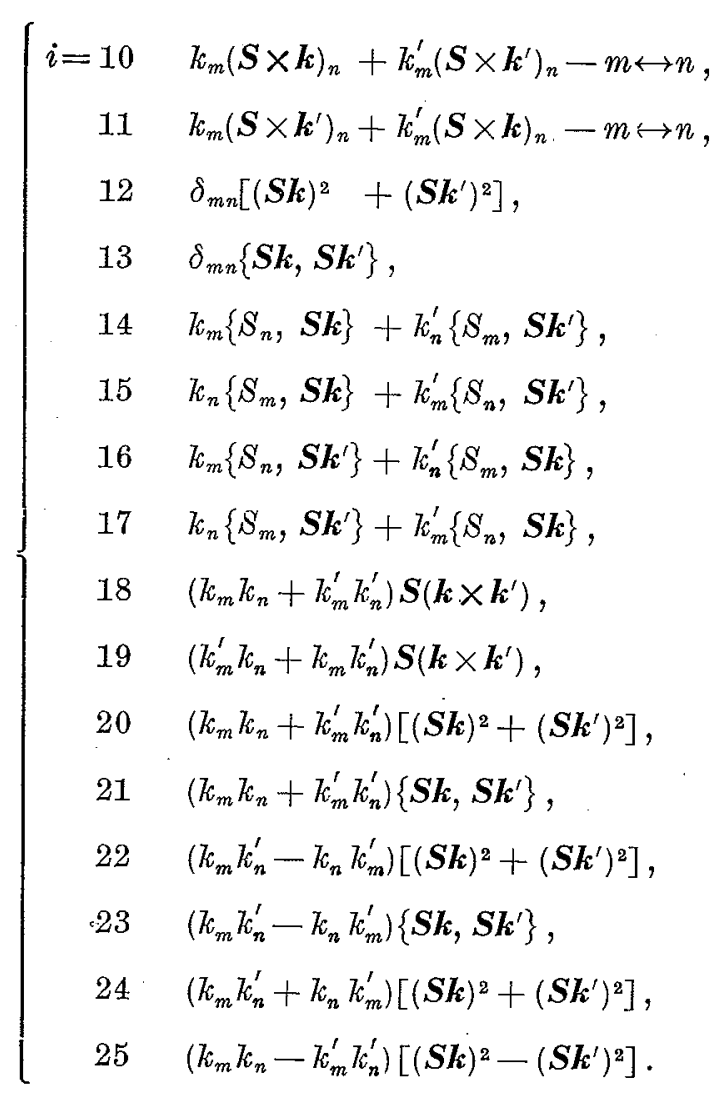

Remarts.

1) The complete minimal basis for spin $\frac{1}{2}$ is obtained by dropping $i=3$, $12, \ldots, 17,20, \ldots, 25$.

2) For $S=1$, there are three basis functions which are $O\left(\omega^{0}\right)$. These correspond to eq. (2.1). There are fourteen functions $O\left(\omega^{2}\right)$; and eight which are $O\left(\omega^{4}\right)$. By tensor analysis it is easy to check that these powers of $\omega$ are indeed minimal.

The general formalism is now complete for the discussion of the low-energy theorems for $S=1$. Observe that every equation in this Section is valid in any reference system. In the discussions now to follow we shall specifically choose the laboratory system.

\section{4. - The case $S=1$.}

For the purpose of computing $U_{\mu \nu}$, eq. (3.6), we must first discuss the $S=1$ electromagnetic vertex, with both $S=1$ particle legs on the mass shell. 
We have

$$
(2 \pi)^{\dot{3}} \cdot\left(4 E^{\prime} E\right)^{\frac{1}{2}}\left\langle p^{\prime} \lambda^{\prime}\left|j_{\mu}\right| p \lambda\right\rangle=\eta_{\varrho}^{\left(\lambda^{\prime}\right)}\left(p^{\prime}\right) X_{\varrho^{\sigma}}^{\mu}\left(p^{\prime}, p\right) \eta_{a}^{(\lambda)}(p)
$$

Here $\lambda, \lambda^{\prime}$ denote polarization states. The functions $\eta_{a}^{(\lambda)}(p)$ may be expressed as follows:

$$
\left\{\begin{array}{l}
\eta^{(\lambda)}(p)=\eta^{(\lambda)}+\frac{p\left(\eta^{(\lambda)} p\right)}{M(E+M)} \\
\eta_{4}^{(\lambda)}(p)=i \frac{\eta^{(\lambda)} p}{M}
\end{array}\right.
$$

where $\eta^{(\lambda)}$ is the polarization vector for $\boldsymbol{p}=0 . X_{\varrho o}^{\mu}$ is given by (11)

$$
\left\{\begin{array}{l}
X_{\varrho^{\sigma}}^{\mu}=\left\{F_{0}\left(q^{2}\right) \delta_{\varrho^{\sigma}}+F_{2}\left(q^{2}\right)\left(q_{\varrho} q_{\sigma}-\frac{q^{2}}{3} \delta_{\varrho \sigma}\right)\right\} P^{\mu}+F_{1}\left(q^{2}\right)\left(\delta_{\mu \sigma} \delta_{\nu \varrho}-\delta_{\nu \sigma} \delta_{\mu \varrho}\right) q^{\mu} \\
P^{\mu}=p^{\mu}+p^{\mu}, \quad q^{\mu}=p^{\mu}-p^{\mu} .
\end{array}\right.
$$

In order to exhibit explicitly the dependence of the vertex on $\boldsymbol{S}$, it is advantageous to "unboost" the $\eta(p)$ functions and write

$$
\eta_{\varrho}^{\left(\lambda^{\prime}\right)}\left(p^{\prime}\right) X_{\varrho^{\sigma}}^{\mu}\left(p^{\prime}, p\right) \eta_{\varrho}^{(\lambda)}(p)=\eta_{\varrho}^{\left(\lambda^{\prime}\right)} Y_{\varrho^{\sigma}}^{\mu}\left(p^{\prime}, p\right) \eta_{\sigma}^{(\lambda)}
$$

We can then write $Y_{e^{\sigma}}^{\mu}$ briefly as $Y^{\mu}$, where the suppressed indices $\varrho, \sigma$ now refer to target spin indices. This gives

$$
\begin{gathered}
\boldsymbol{Y}=\psi \boldsymbol{P}+\frac{F_{\mathbf{0}}(0)}{2 M^{2}} \boldsymbol{P} \varphi+i(\boldsymbol{S} \times \boldsymbol{q}) F_{\mathbf{1}}(0)+O\left(p^{3}\right) \\
-i Y_{\mathbf{4}}=\psi\left(E(\boldsymbol{p})+E\left(\boldsymbol{p}^{\prime}\right)\right)+\left[\frac{\left(E(\boldsymbol{p})+E\left(\boldsymbol{p}^{\prime}\right)\right) F_{\mathbf{0}}}{2 M^{2}}-\frac{F_{1}}{M}\right] \varphi+O\left(p^{4}\right),
\end{gathered}
$$

with

$$
\left\{\begin{array}{l}
\psi=F_{0}-\left\{(\boldsymbol{S} \boldsymbol{q})^{2}-\frac{2}{3} \boldsymbol{q}^{2}\right\} F_{2} \\
\varphi=i \boldsymbol{S}\left(\boldsymbol{p} \times \boldsymbol{p}^{\prime}\right)+\boldsymbol{q}^{2}-(\boldsymbol{S} \boldsymbol{q})^{2}
\end{array}\right.
$$

(11) See V. GLASER and B. JAKŠIĆ: Nuovo Cimento, 5, 1197 (1957); B. SAKITA and C. Goebel: Phys. Rev., 127, 1787 (1962) (Appendix). In these papers an incorreet identification is made of $F_{2}(0)$ with the quadrupole moment. 
The multipole moments are given by ${ }^{(2)}$

$$
\begin{aligned}
& F_{0}(0)=e, \\
& F_{1}(0)=e \mu, \\
& F_{2}(0)=\frac{e}{2 M^{2}}[Q+\mu-1] .
\end{aligned}
$$

Here $\mu$ is the magnetic moment in units $e / 2 M$ and $Q$ is the quadrupole moment in units $e / M^{2}$.

A straightforward calculation of $U_{44}$, see eqs. (3.5), (3.7) yields (for $\boldsymbol{p}=0$ )

$$
\begin{aligned}
& J_{44}=\frac{\psi(\omega) \psi\left(\omega^{\prime}\right)}{M}\left[\cos \theta+\frac{\omega^{2}}{4 M^{2}}\left(1+\cos \theta-2 \cos ^{2} \theta\right)\right]+ \\
& +\frac{e^{2}(1-\mu)}{2 M^{2}}\left[\left\{2 \omega^{2}-(\boldsymbol{S} \boldsymbol{k})^{2}-\left(\boldsymbol{S} \boldsymbol{k}^{\prime}\right)^{2}\right\} \frac{\cos \theta}{M}-i \boldsymbol{S}\left(\boldsymbol{k} \times \boldsymbol{k}^{\prime}\right) \cdot \frac{\omega+\omega^{\prime}}{\omega \omega^{\prime}}\right]+O\left(\omega^{4}\right),
\end{aligned}
$$

where, to the order considered,

$$
\psi(\omega) \psi\left(\omega^{\prime}\right)=F_{0}\left(\omega^{2}\right) F_{0}\left(\omega^{\prime 2}\right)+e F_{2}(0)\left[\frac{4 \omega^{2}}{3}-(S k)^{2}-\left(S k^{\prime}\right)^{2}\right] .
$$

For $U_{m n}$, see eq. (3.8), one finds that

$$
\begin{aligned}
& k_{m}^{\prime} U_{m n} k_{n}=\frac{e^{2}}{4 M^{3}} \omega^{4}(1-2 \cos \theta)+O\left(\omega^{6}\right) \\
& \begin{aligned}
\varepsilon_{m}^{\prime} U_{m n} \varepsilon_{n}=-\frac{e^{2} \mu^{2}}{4 \cdot M^{3}}[i M \omega & \boldsymbol{S}\left(\boldsymbol{\epsilon}^{\prime} \times \boldsymbol{\epsilon}\right)+ \\
& \left.+\boldsymbol{\epsilon}^{\prime} \boldsymbol{\epsilon}\left(\boldsymbol{S}^{2} \omega^{2}-(\boldsymbol{S k})^{2}\right)-\frac{\omega^{2}}{2}\left\{\boldsymbol{S} \boldsymbol{\epsilon}, \boldsymbol{S} \boldsymbol{\epsilon}^{\prime}\right\}\right]+O\left(\omega^{3}\right)
\end{aligned}
\end{aligned}
$$

We are now ready to piece things together and read off the low-energy theorems.

41. Low-energy theorem to $O\left(\omega^{0}\right)$ and $O\left(\omega^{1}\right)$. - We start from eq. (3.9) and note the following.

1) For $U_{4 i}$ we use eq. (4.11) but with eq. (4.12) simplified further to $\psi(\omega) \psi\left(\omega^{\prime}\right) \simeq e^{2}$.

(12) For the standard definition of $Q$ see N. RAMSEY : Nuclear Moments (New York, 1953), p. 16. 
2) We may neglect in eq. (3.9) the term $k_{m}^{\prime} J_{m n} k_{n}$.

3) The same is true for $\omega^{\prime} \omega E_{44}$.

4) We may use eqs. (3.36) and (3.39) with $i=1,2,3$ only.

This gives at once the eqs. (2.3), (2.5), (2.6). To obtain eq. (2.20), we note that there are two contributions to the magnetic moment terms: one from the first term on the right-hand side of eq. (4.14); and one from solving eq. (3.9) for $A_{2}(\omega)$ to $O(\omega)$ which gives a contribution to $E$ (see eq. (3.11)). This last contribution comes from the term $\sim \boldsymbol{S}\left(\boldsymbol{k} \times \boldsymbol{k}^{\prime}\right)$ in eq. (4.11), as inserted in eq. (3.9). The net result is eq. (2.20) with $S=1$.

4:2. The quadrupole-moment theorem. - We shall not give all low-energy theorems that exist to $O\left(\omega^{2}\right)$, but will confine ourselves to the theorem for $Q$.

From eqs. (4.10)-(4.12) we see that $Q$ is associated with a term $\sim\left[(\boldsymbol{S k})^{2}+\right.$ $\left.+\left(\boldsymbol{S k}^{\prime}\right)^{2}\right]$ in ${U_{44}}_{4}$. This indicates how to proceed ( $\left.{ }^{13}\right)$ with eq. (3.9) which we now need to $O\left(\omega^{4}\right)$.

1). From eqs. (4.10)-(4.12) it follows that, for the present purpose, we may put in eq. (3.9)

$$
U_{44} \simeq-\frac{e^{2}}{2 M^{3}} Q \cos \theta\left[(\boldsymbol{S k})^{2}+\left(\boldsymbol{S} \boldsymbol{k}^{\prime}\right)^{2}\right] .
$$

2) It follows from eq. (4.13) that we may neglect in eq. (3.9) the contribution from $k_{m}^{\prime} U_{m n} k_{n}$ because it does not contain the correct spin tensor.

3) In order to get $E_{44}$ in eq. (3.9) to the requisite order, it follows from eq. (3.12) that we may put

$$
\Lambda_{m n}\left(\omega^{\prime}, k^{\prime} ; \omega, k\right) \rightarrow \Lambda_{m n}(0,0 ; 0,0) .
$$

This constant part of $\Lambda_{m n}$ gives a term in $\omega \omega^{\prime} E_{44}$ of the most general form

$$
\simeq \omega^{2}\left[a \boldsymbol{k} \boldsymbol{k}^{\prime}+b\left\{\boldsymbol{S} \boldsymbol{k}, \boldsymbol{S} \boldsymbol{k}^{\prime}\right\}\right],
$$

with constant (and unknown) coefficients $a, b$. It follows from a comparison with eq. (4.15) that these terms have a distinct spin angular dependence from the $Q$ term. Hence $E_{44}$ may be neglected in so far as the $Q$ determination is concerned.

$\left.{ }^{13}\right)$ The kinematical relation $\left(\omega^{\prime}-\omega\right) M=\omega \omega^{\prime}(\cos \theta-1)$ is often used in what follows. 
4) In regard to $E_{m n}$, we use eqs. (3.36) and (3.39). To $O\left(\omega^{2}\right)$ in $E_{m n}$ we may drop the terms with $i=18, \ldots, 25$. It will readily be checked that the pertinent terms are $i=12$ and $i=14$. Inserting all this into eq. (3.9) one finally arrives at the low-energy theorem

$$
A_{12}(0)+2 A_{14}(0)=\frac{e^{2} Q}{M^{2}} .
$$

We must finally translate this information in the language of eq. (2.7). It is easily seen that the $Q$ terms correspond to $\sim \omega^{2} Y_{2 M}(\theta, \phi)$ in eq. (2.7). The final answers are as follows. Put

$$
A^{(N L M)} \equiv A_{11}^{(N L M)}+A_{\mathbf{2 2}}^{(N L M)}
$$

Then

$$
\begin{aligned}
& A^{(22 \pm 2)}=4 \sqrt{\frac{2 \pi}{15}} e^{2} Q S_{ \pm}^{2}, \\
& A^{(22 \pm 1)}=\mp \sqrt{\frac{8 \pi}{15}} e^{2} Q\left\{S_{\mp}, S_{3}\right\}, \\
& A^{(220)}=-\frac{1}{3} \sqrt{\frac{4 \pi}{5}} e^{2} Q\left(S^{2}-S_{3}^{2}\right), \\
& 2 S_{ \pm}=S_{1} \pm i S_{2} .
\end{aligned}
$$

Remarks. 1) The preferred nature of the three-direction in eqs. (4.19)-(4.22) is simply due to the choice of $\boldsymbol{k}$-direction. 2) The eqs. (4.20)-(4.22) are spin matrix equations. For the case of eq. (4.22) the following stipulation is needed: valid for all spin transitions except $S_{3}=1 \rightarrow S_{3}=1$; and $S_{3}=-1 \rightarrow S_{3}=-1$. Of course these two transitions are equal. The fact that one element of $A^{\{20\}}$ remains undetermined is because, due to the spin independence of the trace of $A^{(220)}$, low-energy theorems cannot determine this trace.

\section{5. - The case of general spin.}

Under this heading we shall only discuss the following topics.

51. The validity of the three zero-energy theorems. - This is of course elementary. Equation (2.5) follows from crossing symmetry, for any $S$. To get eqs. (2.3), (2.6), use eq. (3.9) to $O\left(\omega^{2}\right)$. To this order neither the $E_{44}$ nor the $D_{m \cdot n}$ term in that equation contributes. To the order considered, the $E_{m n}^{(i)}$ of eq. (3.39) with $i=1,2,3$ form a complete basis for any $S$. Equations (2.3), 
(2.6), then are consequences of

$$
(2 \pi)^{3}\left\langle 0 \lambda^{\prime}\left|j_{4}\right| 0 \lambda\right\rangle=i e \delta^{\left(\lambda^{\prime} \lambda\right)}
$$

where notations are similar to eq. (4.1) except that the range of $\lambda, \lambda^{\prime}$ is from 1 to $2 S+1$. Inserting eq. (5.1) into $U_{44}$ gives the Thomson limit for $A_{1}(0)$ and the vanishing of $A_{3}(0)$.

52. The low-energy magnetic-moment theorem for any $S$. - The question is to show that eq. $(2.20)$ is valid for any $S$. It is known $\left({ }^{2.3}\right)$ to be true for $S=\frac{1}{2}$; and the derivation for $S=1$ was given in Sect. 4 , see also ref. $\left({ }^{8}\right)$.

We start from the general definition of the magnetic moment operator

$$
\mathscr{H}=\frac{1}{2} \int \boldsymbol{r} \times \boldsymbol{j}(x) \mathrm{d}^{3} x
$$

By the usual method $\left({ }^{14}\right)$ it follows that the one-particle state matrix elements at zero momentum are given by

$$
\left.\left\langle 0, \lambda^{\prime}|\mathscr{M}| 0, \lambda\right)\right\rangle=\frac{i}{2} \cdot(2 \pi)^{3} \lim _{\boldsymbol{p}=0} \lim _{\boldsymbol{p}^{\prime}=\boldsymbol{p}}\left[\nabla_{\eta} \times\left\langle\boldsymbol{p}^{\prime} \lambda^{\prime}|\boldsymbol{j}| \boldsymbol{p}, \lambda\right\rangle\right]
$$

To first order in the momenta

$$
\left\langle\boldsymbol{p}^{\prime} \lambda^{\prime}|\boldsymbol{j}| \boldsymbol{p}, \lambda\right\rangle=\frac{1}{(2 \pi)^{3}} \frac{e \mu}{2 M S} \cdot i(\boldsymbol{S} \times \boldsymbol{q})
$$

with $\boldsymbol{q}=\boldsymbol{p}^{\prime}-\boldsymbol{p}$. Thus the gyromagnetic ratio $g=\mu / S$ enters from the outset through the structure of the current matrix element. The same is true for a term of second order in the momenta contained in the matrix element of $j_{4}$ :

$$
\left\langle\boldsymbol{p}^{\prime}, \lambda^{\prime}\left|\boldsymbol{j}_{a}\right| \boldsymbol{p}, \lambda\right\rangle \text { contains a term } \cdot \frac{1}{(2 \pi)^{3}} \cdot \frac{e}{2 M^{2}}(g-1)\left\langle\lambda^{\prime}|\boldsymbol{S}| \lambda\right\rangle \cdot\left(\boldsymbol{p} \times \boldsymbol{p}^{\prime}\right) .
$$

This information is sufficient to compute eq. (2.20). The $U$ term in eq. (3.10) gives a contribution which is found from eqs. (3.8) and (5.4). The $E$ term in eq. (3.10) gives a contribution which comes from the basis function $E_{m n}^{(2)}$ in eq. (3.39). One inserts this function into eq. (3.9), observes that $E_{44}$ and $k_{m}^{\prime} U_{m n} k_{n}$ do not contribute and that, to the order considered, the contribution of $U_{44}$ arises from the cross-terms between eqs. (5.1) and (5.5), inserted into eqs. (3.5), (3.7).

(14) F. J. ERNST, R. G. SACHS and K. C. WALI : Phys. Rev., 119, 1105 (1960). 
5.3. -The general intrinsic multipote theorem states $\left({ }^{1}\right)$ that there exists a low-energy theorem for any of the $2 S+1$ intrinsic multipole moments of a stable particle with spin $S$. The strategy of this existence proof is the following. Start from eq. (3.9) and determine the spin and orbital multipole structure of the terms in $U_{\mu \nu}$ which are potential candidates for a low-energy theorem. Insert those terms in eq. (3.9) and verify that, for these terms, $E_{44}$ in that equation plays no role. As soon as it is clear that $E_{44}$ may be dropped, the existence of the low-energy theorem is proved. The actual evaluation of such higher multipole theorems then follows along the lines of Sect. 4 for $Q$. In particular one must extend the minimal base for $E_{m n}$ to the appropriate spin value.

$5^{*} 4$. Electric $2^{L}$ pole moments, $(L$ even). - To these moments one associates the operator: $\left.{ }^{15}\right)$

$$
Q^{(2 L)} \sim \int x_{n_{1}} x_{n_{2}} \ldots x_{n_{2 L}} \varrho(x) \mathrm{d}^{3} x
$$

where $o$ is the electric charge density. $Q^{(2 L)}$ is not the $2^{L}$ operator itself. To get the latter one has to take out $\left(^{16}\right)$ all traces from $Q^{(2 L)}$. The Fourier transform of $Q^{(2 L)}$ will make its appearance in a form factor $F_{2 L}$ in the electromagnetic vertex. In $U_{44}$, a term $\sim e F_{2 x}(0)\left[\left\langle k^{2 L}\right\rangle+\left\langle k^{\prime 2 L}\right\rangle\right] \cos \theta$ will appear (cf. eqs. (4.11), (4.12), (4.15) for $L=1$ ) where $F_{2 L}(0)$ contains the $2^{L}$ moment ( $\left.{ }^{17}\right)$. Therefore,

$$
\omega^{\prime} \omega U_{44} \text { contains } e F_{2 L}(0)\left\langle k^{\prime 2 L}\right\rangle \cdot k k^{\prime}
$$

Now consider $E_{44}$. To be in competition, only terms containing $2 L$ factors $S_{i}$ $\left(i=1,2\right.$ or 3 ) are relevant. Thus the only terms of $\Lambda_{m n}$, see eq. (3.12) which have to be considered are

$$
\sum_{n=0}^{2 L-2} c_{n} \sum_{P} S_{m} S_{n}(\boldsymbol{S} \boldsymbol{k})^{2 L-2-n}\left(\boldsymbol{S} \boldsymbol{k}^{\prime}\right)^{n}
$$

where the $P$ summation is over all permutations of $S$ factors. The $c_{n}$ are

${ }^{\left({ }^{15}\right)}$ J. M. BlatT and V. F. WeIsskopf: Theoretical Nuclear Physics (New York, 1952), p. 807.

(16) As was seen after eq. (4.22) for the case $L=1$, the use of reducible tensors $Q^{(2 L)}$ brings with it that a few of the multipole transition elements remain undetermined, without, however, impairing the existence of a low-energy theorem for the remaining transition elements.

${ }^{(17)}$ See the notation defined in eq. (3.28), $F_{2 L}(0)$ will also contain lower multipole moments. In the sense of a proof by induction, we assume that these are already fixed by their lower-moment theorem. 
constants (to our order) because it follows from eq. (3.12) that the terms (5.7) contribute to the same order in $\omega$ to $\omega \omega^{\prime} E_{44}$ as is the order $(2 L+2)$ which appears in eq. (5.6). We now look for the highest orbital multipole which appears in eq. (5.7), i.e., for the highest possible power in $\boldsymbol{k}^{\prime}$. This yields a term in $\omega \omega^{\prime} E_{44}$

$$
\sim \omega^{2}\left\langle k\left(k^{\prime}\right)^{2 L-1}\right\rangle
$$

The highest orbital multipole contained herein is $\sim \omega^{2 L+2} Y_{2 L-1, M}$. It follows that $E_{44}$ cannot compete in the determination from eq. (3.9) of terms $\sim \omega^{2 L+2} Y_{2 L+1, \pi L}$ which appear in $\omega \omega^{\prime} U_{44}$, according to eq. (5.6). Hence $E_{44}$ may be dropped which is what we need. (Note: the way in which $k_{m}^{\prime} U_{m n} k_{n}$ contributes in eq. (3.9) need not be considered explicitly.)

5.5. Magnetic $2^{L}$ pole moments $\left(L\right.$ odd). - To the $2^{2 L+1}$ pole one associates the operator $\left({ }^{15}\right)$

$$
\mathscr{M}^{(2 L)} \sim \int x_{n_{1}} x_{n_{2}} \ldots x_{n_{2 L}}(\boldsymbol{r} \times \boldsymbol{j}(x)) \mathrm{d}^{3} x
$$

With similar comments as for the electric case we isolate the highest orbital multipole term in $\omega \omega^{\prime} U_{44}$ which is

$$
\sim e F_{2 L+1}(0)\left\langle k^{\prime 2 L} R\right\rangle\left(\omega^{\prime}+\omega\right)
$$

using the notations of eqs. (3.18), (3.28). (Note that a distinct behaviour in the powers of $\omega$ and $\omega^{\prime}$ comes from the opposite crossing properties of the magnetic as compared to the electric terms.) $F_{2 L+1}(0)$ contains the $2^{2 L+1}$ pole moment. By the same method as for the electric case one checks that $\omega \omega^{\prime} E_{44}$ cannot compete. This completes the proof of the multipole moment theorem.

I want to express my gratitude to the CERN for its generous hospitality.

\section{RIASSUNTO $\left(^{*}\right)$}

Per scattering di Compton su particelle stabili di spin $S$ esiste, a prescindere dalle regole di somma di Thomson e quella di $(g-2)$, un insieme di $(2 S-1)$ (per $S \geqslant 1$ ) regole di somma per sezione d'urto totale; regole di somma che, in forma non sot-

(*) Traduzione a cura della Redazione. 
tratta, sono relazioni di superconvergenza. Applicata a nuclei, la possibile validità di questa forma di non sottrazione può essere collegata all'additività asintotica. Si dimostra l'esistenza di teoremi di bassa energia per tutti i $2 S+1$ momenti di multipolo intrinseci. Si considera particolarmente il caso in cui $S=1$.

\section{Комптоновское рассеяние на стабильных мишенях с произволыным спином.}

Резюме $\left(^{*}\right) .-$ - Для комптоновского рассеяния на стабильных частицах со спшном $S$ существует, назависимо от томсоновских и $(g-2)$ правил сумм, система $(2 S-1)$ правил сумм для попных поперечных сечений (для $S \geqslant 1$ ), которая, в безвычитательной форме, представляет сверхсходящиеся соотношения. Будучи примененной $\mathrm{k}$ ядрам, возможғая справедливость этой безвычитательной формы может быть связана с асимптотической аддитивностью. Доказывается, что теоремы низких энергий существуют для всех $2 S+1$ внутренних мультипольных моментов. Подробно рассматривается случай $S=1$.

(*) Переведено редакцией. 